\title{
Educación y Participación Comunitaria en Escuelas de Contulmo y Tirúa, Chile. Interculturalidad, Micropolíticas y Territorios
}

\section{Education and Community Engagement in Schools in the Communities of Contulmo and Tirua, Chile. Interculturality, Micropolitics and Territories}

\author{
Inti Torres Villegas * \\ Noelia Carrasco Henríquez \\ Universidad de Concepción
}

\begin{abstract}
El presente artículo describe tres factores que favorecen el vínculo entre propuestas educativas y procesos de participación comunitaria en comunidades Mapuche en Chile. A través del análisis de diversas experiencias se revela una resignificación progresiva de las escuelas, situándolas como espacio de participación y confluencia colectiva, en donde se manifiestan diversas tensiones y acuerdos presentes al interior de las comunidades donde se localizan, posicionándolas como puntos estratégicos para la definición y la apropiación territorial. Se profundiza en el análisis de la interrelación entre escuela y comunidad, a partir de identificar y caracterizar cuales son los factores que la potencian e inhiben, con la finalidad de identificar como operan y de qué forma son percibidas las escuelas al interior de las comunidades Mapuche en las comunas arriba mencionadas. Para lo anterior, se recogieron múltiples testimonios aportados por integrantes de tres escuelas participantes y de personajes de referencia de las comunidades donde estas se localizan, los cuales fueron analizados cualitativamente, lo que permitió identificar diversas mecánicas de participación comunitaria incidiendo, en pequeña escala, en el diseño y ejecución de propuestas educativas con pertinencia cultural y territorial, posicionando a las escuelas como espacios de confluencia comunitaria..
\end{abstract}

Descriptores: Educación intercultural, Participación comunitaria, Derechos humanos, Escuela, Diversidad cultural, Cultura mapuche.

\begin{abstract}
This article describes three factors that foster the link between educational models and community engagement processes in Mapuche communities in Chile. In this context, the article reveals, through the analysis of various experiences, how communities have done a progressive re-signification of the schools: defining them as a space for participation and collective confluence, where diverse community tensions and agreements are manifested; positioning them as strategic nodes for the definition and appropriation of the territory. This allows us to deepen the analysis of the relationship between schools and communities, by identifying and characterizing the factors that enhance and inhibit it, in order to identify how they operate and how schools are perceived within the Mapuche communities in the communes mentioned above. Several testimonies were collected from members of the three schools that participated and other communities' stakeholders. These testimonies where analyzed qualitatively, which allowed the identification of diverse community engagement dynamics that affect, on a small scale, in the design and execution of educational proposals with cultural and territorial relevance, positioning the schools as spaces of community confluence.
\end{abstract}

Keywords: Intercultural Education, Community participation, Human rights, School, Cultural diversity. Mapuche culture.

*Contacto: intitorres@udec.cl

ISSN: 0718-7378

www.rinace.net/rlei/ $\begin{array}{ll}\text { Recibido: } & 19 / 05 / 2017 \\ 1^{a} \text { Evaluación: } & 15 / 09 / 2017 \\ \text { Aceptado: } & 02 / 10 / 2017\end{array}$ 


\section{Introducción}

Los procesos educativos cultural y territorialmente situados, han jugado un rol fundamental en la apertura de espacios de reflexión para la transformación social. En ese sentido, podríamos identificar también a los procesos educativos, como elementos de suma importancia en la construcción cotidiana de identidades, individuales y colectivas, lo que ubicaría a la educación como un espacio social clave en las construcciones políticas locales. Dado lo anterior, la educación no puede interpretarse como una dinámica aislada de otros procesos sociales, sino como un espacio donde estos confluyen y se manifiestan, evidenciando relaciones de poder, consensos y contradicciones, acuerdos y tensiones, dinámicas de cohesión o también de fragmentación comunitaria. Lo anterior, invita a la reflexión del proceso educativo, entendiéndolo como un espacio en permanente disputa entre diversos actores sociales que operan al interior de las comunidades, el cual, en muchos casos ha decantado en modelos educativos estandarizados, con poca pertinencia cultural y territorial, y que privilegian lo institucional por sobre lo comunitario, invisibilizando saberes y dinámicas locales para la construcción de conocimientos y prácticas.

Históricamente, los pueblos originarios de América Latina han sido objeto de violación a sus derechos, lo cual se ha materializado no solo en el despojo progresivo de sus tierras y en la sistemática negación del acceso a la justicia, sino también en la imposibilidad de incidir en la definición del tipo de educación que se imparte dentro de sus comunidades. La suma de todo lo anterior, sitúa a los pueblos originarios en el continente americano en una alarmante situación de marginación y dependencia política y cultural. En gran medida, la situación actual de los pueblos originarios, encuentra raíz en la negación histórica que los Estados Nacionales en la región han hecho de sus dinámicas particulares de organización, participación y transmisión de conocimiento. En el caso particular del pueblo Mapuche, resulta imperante admitir que históricamente el Estado Chileno no ha sido capaz de generar transformaciones que contribuyan substancialmente a la autodeterminación Mapuche en aspectos organizativos, participativos o educativos, sino que, por el contrario, ha promovido a través de diversos medios, un carácter de unicidad nacional a pesar, y en contra de, la rica diversidad cultural presente en el territorio nacional. Así lo aseguró el Informe de la Comisión Verdad Histórica y Nuevo Trato $^{1}$ en 2003, cuando menciona que basta un análisis de los marcos jurídicos en el país para entender la concepción unitaria del Estado de Chile, y que por lo mismo el término pueblo se ha asumido como el de: distintos grupos que forman parte del Estado, pero no como naciones. Esta premisa se ha traducido en la construcción de una política pública que, si bien ha avanzado en el reconocimiento progresivo de los pueblos originarios presentes en el territorio nacional, aún resulta insuficiente para la generación de procesos autónomos y de autodeterminación de los mismos.

Enmarcado en lo anterior, destaca la creación en 1995 de una iniciativa de acción dentro del Programa de Educación Básica Rural al que correspondería desarrollar una serie proyectos pilotos en educación intercultural bilingüe en escuelas y liceos con población indígena, en conjunto entre la Corporación Nacional de Desarrollo Indígena (CONADI) y el Ministerio de Educación (MINEDUC). Este proceso desencadenaría en la posterior 
creación del Programa de Educación Intercultural Bilingüe (PEIB) que en el año 2000 se institucionaliza como programa independiente y se comienza a aplicar como una estrategia de focalización y expansión paulatina a establecimientos con características similares a las participantes de los pilotos anteriormente mencionados (MINEDUC, 2011). A partir de ahí, las líneas centrales del PEIB quedaron definidas en torno a diversos objetivos que principalmente buscaron, por un lado, la formación y capacitación de profesores y especialistas en interculturalidad y bilingüismo, y por otro, la generación de propuestas curriculares con pertinencia cultural y territorial. En consideración de lo anterior, es importante destacar que aunque el PEIB considera la inclusión de educadores tradicionales, quienes como integrantes de la comunidad donde se encuentra inserta la escuela, apoyan para incorporar conocimientos de la cultura originaria a las prácticas educativas, no describe en sus normativas un mecanismo especifico de consulta que sea vinculante $^{2}$ a través de la cual las comunidades puedan tener una incidencia concreta en el diseño de planes y programas particularizados cultural y territorialmente. Lo anterior, invita a preguntarnos por las características del diseño y la ejecución institucional del PIEB, y también por el impacto político y cultural real que su propuesta educativa puede generar dentro de las comunidades donde se aplica. Cabe indicar que las propias directrices del PIEB señalan que éste debe tener un rol activo en el fomento de la participación de la familia y la comunidad en los procesos de enseñanza-aprendizaje (ob cit, p.10). Por tanto, esta divergencia entre el discurso y la práctica abre nuevas interrogantes sobre el tipo de participación que desde la institucionalidad escolar se promueve, y sobre si esta ofrece una alternativa real de decisión para el pueblo Mapuche en cuanto a los conocimientos que se consideran relevantes transmitir y construir en conjunto con las nuevas generaciones. Este cuestionamiento incluye también preguntas respecto a la función misma de las escuelas dentro de las comunidades Mapuche, que lo mismo podrían asumirse como espacios de confluencia comunitaria que como elementos ajenos y disruptivos de la tradición local, según la experiencia de las y los participantes del proceso educativo.

No obstante, a pesar de las carencias estructurales del PEIB anteriormente señaladas, resulta relevante admitir que muchos han sido los esfuerzos efectivos por vincular a las escuelas operando bajo esta modalidad con las comunidades donde se localizan, impulsados por las y los integrantes de diversas comunidades escolares, quienes, paralelamente a las directrices del PEIB, impulsan iniciativas pedagógicas y de participación escolar, consolidando una serie de experiencias positivas que han impactado favorablemente no solo el trayecto escolar de las y los estudiantes, sino en general la experiencia de todas y todos los participantes del proceso educativo. Como se expondrá con mayor detalle más adelante, estos esfuerzos se gestan mayormente a nivel de escuela y comunidad, logrando también un impacto mayor en la progresiva construcción de política educativa a nivel institucional. A pesar de esto, y en voz de las y los protagonistas de estas iniciativas, pocos han sido los esfuerzos institucionales por sistematizar estas experiencias de vinculación entre escuela y comunidad, en contraste con el desmedido esfuerzo por calificar cuantitativamente aspectos como el desempeño académico de las escuelas a través de pruebas estandarizadas.

2 Según el sitio web del MINEDUC. durante el año 2010 y 2011, el Ministerio a través del Programa Intercultural Bilingüe, realizó la consulta a los pueblos indígenas, a propósito del Decreto que regula la función docente de Educadores (as) Tradicionales que implementan la Lengua Indígena en establecimientos del país. Información disponible en: http://portales.mineduc.cl/index2.php?id_portal=28\&id_seccion=5418\&id_contenido=33789 
En consonancia con los antecedentes arriba señalados, el presente artículo busca relevar aquellos elementos que potencian el impacto recíproco en la interrelación entre educación y procesos de participación comunitaria, a través de aspectos como la definición identitaria, la apropiación territorial y la movilización colectiva, que en suma pueden derivar en pautas dinámicas de acción comunitaria construidas y desarrolladas desde las escuelas. Lo anterior se enmarca dentro de la investigación Interrelación entre procesos de educación y participación comunitaria en contexto Mapuche: Tres experiencias en el cono sur de la Provincia de Arauco presentada como tesis del magister de Investigación Social y Desarrollo de la Universidad de Concepción, la cual se realizó durante Noviembre de 2016 y Abril de 2017, a partir de la recuperación de la experiencia de tres colegios municipales - dos en la comuna de Contulmo y uno en la comuna de Tirúa- que implementan el PEIB dentro de la provincia de Arauco, en la Región del Bio Bio. Es importante mencionar que la elección de estas dos comunas no resultó en absoluto casual pues según el Instituto Nacional de Estadística (INE, 2005) en el último censo realizado en 2002 señala que ambas son dos de las tres con mayor porcentaje de población Mapuche en la Región del Bio-Bio y que incluso Tirúa es la 5ta en el país. Por otro lado, también cabe señalar que el cono sur de la Provincia de Arauco, donde se localizan las escuelas participantes, ha sido escenario de números conflictos sociales y territoriales principalmente entre el Estado Chileno, las empresas forestales y las comunidades Mapuche, lo cual constituye un contexto complejo de relevancia para el análisis de las temáticas expuestas previamente.

\section{Revisión de la literatura}

La discusión conceptual sobre la interrelación entre la educación y la participación comunitaria, buscará relevar qué factores potencian que este vínculo pueda generar, dentro de los contextos particulares en los cuales se desarrolla, procesos concretos de movilización social. Lo anterior, no implica necesariamente dejar de lado los aspectos pedagógicos, ni mucho menos los relacionados con la construcción de política educativa, sino más bien reflexionar sobre cómo estos dos aspectos, desarrollados de forma inclusiva, participativa, y con pertinencia cultural y territorial, pueden potenciar a la educación como espacio para la reflexión, el dialogo y la acción social. Así mismo, permite indagar en cómo procesos de participación comunitaria, en distintas escalas y en diferentes ámbitos, pueden decantar en la construcción de propuestas educativas con las características arriba enunciadas.

En el ámbito educativo, son fácilmente identificables las posturas que conciben a la educación no solamente como un proceso para el desarrollo individual de habilidades, sino como un espacio para el fortalecimiento colectivo de las capacidades. En particular, Mauricio Huircan (2010) describe que en los modelos de educación tradicional Mapuche “dominar saberes y conocimientos genera ciertos beneficios para su entorno próximo, vinculado con la mantención del equilibrio y la existencia armoniosa dentro de la comunidad" (p. 25). Este planteamiento, se contrapone con modelos educativos orientados exclusivamente a la formación individual de competencias, donde se priorizan los objetivos de una economía de mercado, interesada en que las instituciones educativas se transformen en centros de adiestramiento laboral, ofertando tan solo una porción de conocimiento y las destrezas individuales suficientes para aceptar empleos precarios y mal pagados (Llamas, 2014). Una educación meramente enfocada a la capacitación 
laboral individual, privilegia contenidos estandarizados, mayormente técnicos, desconociendo o menospreciando el cúmulo de saberes y prácticas de transmisión de conocimientos propias de los pueblos originarios. En ese sentido, Rojas y Peña (2015), afirman en lo respectivo a los procesos de sociabilización del conocimiento de las y los estudiantes de comunidades Mapuche, que la forma en la que está definida la educación oficial y sus contenidos conlleva a un problema para las comunidades, ya que desde las escuelas se ha negado históricamente otro tipo de conocimiento que no sean el occidental, en detrimento de aspectos como la narración oral y el conocimiento sobre las relaciones de parentesco entre los Mapuche (Rojas y Peña, 2015). Lo anteriormente expuesto, además de identificar dos énfasis en la comprensión de la educación, a saber, la individual y la colectiva, plantea la interrogante sobre ¿cómo construir modelos educativos que potencien el sentido de colectividad dentro de los contextos en los que se desarrollan, en lo que respecta a la selección de contenidos y estrategia pedagógica? Una propuesta al respecto se desprende del enfoque de Derechos, descrito por Luna (2012), cuando menciona que el sentido de la educación y sus conceptos de calidad tienen que nutrirse de la doctrina de los Derechos Humanos y del entendimiento de que la educación es un derecho fundamental que transversaliza a todos los derechos. Lo anterior, señala, "establece una interrelación de la educación con todos los factores y dimensiones de la realidad en distintos niveles. Y también en la gestación de visiones y políticas para la construcción del rumbo de acción de las comunidades" (Luna, 2012, p. 56). Por otro lado, es importante recalcar que el derecho a la educación es simultáneamente individual y social (Nogueira, 2008, p. 210), considerando no solo su importancia en el trayecto de un individuo como medio para lograr una mejor calidad de vida, sino también su relevancia como proceso de preservación cultural y patrimonial para los pueblos. En ese sentido, es necesario entender la educación como un espacio no solo de análisis crítico sino también de construcción de la realidad. En lo práctico, la educación con el enfoque de derechos humanos, no consiste simplemente en la difusión de los marcos jurídicos que garantizan los derechos en diversos niveles, sino también el análisis de cómo un grupo en particular recibe o es vulnerado en sus derechos. En contexto Mapuche, lo anterior supondría no solo un análisis crítico de la historia en la relación entre el pueblo Mapuche y otros actores sociales, políticos y económicos, sino también sobre su coyuntura actual, con el objetivo de generar espacios de reflexión y construcción de propuestas comunitarias de acción. Considerando la compleja y diversa realidad de las comunidades Mapuche, es importante considerar que Crespo (2012: p.71), asume que a la par de su rol como dinámica de transformación social, la educación puede implicar un punto de encuentro, diálogo y puesta en común de la diversidad social de las sociedades cuando afirma que el desafío de la educación apunta a la inclusión de toda la población y a la provisión de una educación de calidad para todos. Pero, además, la educación constituye un espacio público para el ejercicio de la ciudadanía, donde los diversos grupos sociales aprenden a reconocerse y a convivir desde la diversidad.

Asumida la relevancia de la educación ante diversos procesos sociales, convendrá identificar algunas posturas teóricas asumidas ante los desafíos que la diversidad presenta en distintos ámbitos de la realidad y el modo en que éstos son o pueden ser asumidos desde la práctica educativa. En primer lugar, la multiculturalidad, definida por Venegas (2012), consiste en el reconocimiento de la diversidad de culturas conjuntas en un mismo espacio o tiempo, sin considerar la posibilidad de interacción horizontal entre 
las mismas o de un cambio en el tipo de relación histórica entre ellas. El mismo Venegas (2012) sostiene que,

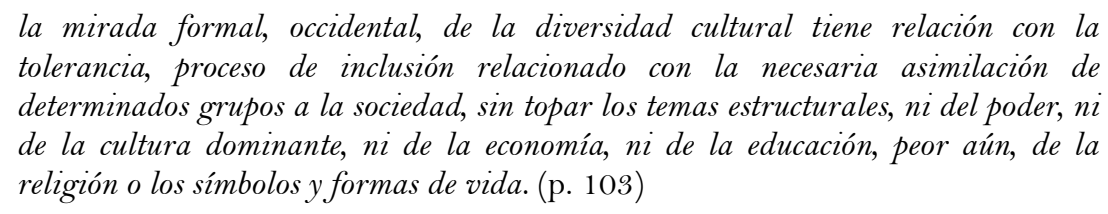

Es decir que, bajo esta lógica, las políticas multiculturales en diversos ámbitos reconocerían la diversidad, pero buscando homogeneizarla hacia la clase o cultura dominante. Basta una breve recapitulación histórica para identificar que, cuando menos en América Latina, históricamente la gran mayoría de los sistemas educativos públicos se orientaron, a través de sus políticas educativas y prácticas pedagógicas, a la estandarización de contenidos, como estrategia para promover la unicidad nacional. El Estado Chileno y su relación con los pueblos originarios presentes en su territorio nacional, no ha sido la excepción.

Por otro lado, el mismo Venegas (2012) sostiene que, por el contrario, "la interculturalidad implica una interrelación horizontal y critica entre dos o más culturas, las cuales, a su vez, se van conjugando y potenciando sistemáticamente" (p. 103). En ese sentido, podría asumirse de antemano que la interculturalidad no solo posibilita el dialogo entre diversos grupos sociales, sino que también potencia una integración que no resigna características o elementos de una cultura ante la otra, ya que, por el contrario, promueve una integración equitativa. Esta definición, es coincidente con lo señalado por Ferrão (2010), recuperando los postulados de Fidel Tubino al respecto, cuando caracteriza a la interculturalidad critica, la cual no solo reconoce las diferencias, sino que cuestiona las desigualdades construidas a lo largo de la historia entre diferentes grupos humanos. Más allá de esta crítica, este tipo de interculturalidad pretende ser una propuesta ética y política para la construcción de sociedades democráticas que articulen "la igualdad y el reconocimiento de las diferentes culturas, y de proponer alternativas al carácter monocultural occidentalizante, que domina en la mayoría de los países del continente latinoamericano" (Ferrão, 2010, p. 338).

Considerando las anteriores propuestas conceptuales, y tal y como se mencionó líneas arriba, es posible advertir que el sistema educativo en Chile, a pesar de ofrecer una propuesta educativa intercultural, amparadas en la Ley Indígena 19.253 y expresada en el PEIB (Huircan, 2010, p. 98), únicamente apunta hacia el reconocimiento multicultural, ya que "en su estructura institucional mantiene carácter centralizado, proveniente de organismos creados por el Estado, y donde se toma como base el conocimiento occidental fundamentado en criterios de objetividad enajenante" (p. 100). En consecuencia, el currículum oficial en Chile reproduce el modelo escolar occidental, por el que han transitado muchas generaciones de estudiantes Mapuche, a partir de la consolidación de la escuela como único dispositivo educativo, el cual responde al imaginario nacional, que si bien ha incluido progresivamente a diversos sectores de la sociedad nacional, continua generando una relación hegemónica del saber occidental en relación con el saber Mapuche.

En síntesis, en materia de educación intercultural, se podría concluir que un modelo educativo que se vincule a procesos de participación comunitaria, requerirá caracterizarse por promover una educación que fortalezca capacidades colectivas, contribuyendo con ello a la cohesión y movilización comunitaria. Por otro lado, deberá 
fundamentarse en un enfoque de derechos humanos, que no solo promueva su ejercicio, sino que también analice de forma crítica su entorno en relación al cumplimiento o vulneración de los derechos colectivos e individuales, para contribuir a la exigencia y la reivindicación de los mismos. Por último, deberá reconocer la diversidad de forma crítica, analizando las relaciones inequitativas históricas entre grupos diversos en tiempos y espacios determinados. En ese sentido, y considerando las características del sistema educativo en Chile, resulta fundamental no solo una revisión crítica de los planes y programas oficiales propuestos por el Huircan (2010) sino también focalizar esfuerzos en identificar y resignificar prácticas cotidianas dentro de las escuelas, que pueden en su ejercicio corresponderse con los conceptos anteriormente expuestos. Esto podría contribuir, a través de la experiencia cotidiana, a que la educación resulte ser motor que impulse transformaciones a diversas escalas y en múltiples ámbitos, para revertir la historia de marginación, segregación y violencia en contra del pueblo Mapuche, que desde diversos sectores sociales se ha ejercido. Para lo anterior, resulta indispensable la identificación de dos ámbitos, o niveles, del quehacer educativo; "el micropolitico y el macropolítico" (Mella, 2010, p. 29), el primero contenido dentro de las escuelas, las aulas, las y los actores del proceso educativo y la comunidad que los rodea, y el segundo relacionado con las variables estructurales, institucionales y normativas del proceso educativo a nivel nacional. Cabe destacar, que ambos niveles no pueden ser vistos como dos espacios aislados que operan con independencia, sino que necesariamente deben comprenderse como dos dimensiones de una misma construcción intersubjetiva y sociocultural. Esta mirada compleja, permite reconocer los alcances concretos del ámbito micropolitico en los diversos trayectos educativos, y su trascendencia en la construcción de propuestas educativas para la trasformación social. Después de todo, los procesos educativos, a pesar de desarrollarse insertos en una estructura superior, institucional y jurídica, son definidos cotidianamente por sus protagonistas a través de esfuerzos individuales y colectivos, que en este nivel guardan vínculos estrechos con las comunidades y los territorios que les rodean. Es decir, los procesos educativos diseñados y supervisados desde espacios de macropolítica operan en el nivel micropolitico, dentro de las escuelas y las comunidades, y desde allí, han de abrir espacios para que las comunidades canalicen sus inquietudes y se retroalimenten internamente.

En lo que respecta al sentido de identidad, coincidimos con García (2014), cuando afirma que "el punto de partida de la conciencia de sí de cualquier ser humano es su identidad. Es decir, la identidad es nuestra afirmación en y hacia el mundo" (García, 2014, p. 9). En, porque define dinámicamente las configuraciones individuales y colectivas y hacia, porque traza esquemas de acción en la construcción de los contextos sociales, geográficos o temporales en los que se habita. Lo anterior, da luz inicial a entender la configuración identitaria como elemento que aglutina y potencia diversos procesos de movilización social, cuestión que permite argumentar la importancia de superar la comprensión de la educación como un proceso de transferencia de conocimientos y promoverlo como un espacio social clave en la construcción de las identidades. Esta dinámica, se encuentra aún presente en las comunidades Mapuche, donde la construcción de conocimientos contribuye a la definición identitaria, promoviendo actitudes y valores como el respeto, el cual se considera como un elemento importante para las interacciones en que se entregan los conocimientos de la cultura (Quintriqueo y Cárdenas, 2010).

Por último, es importante mencionar que no necesariamente la identidad individual fortalecida y focalizada territorialmente deriva irremediablemente en acción colectiva, 
pero es posible que la puesta en común de intersubjetividades compartidas, es decir, de identidades colectivas, confluyan en la construcción de objetivos en común. En ese sentido García (2014), sostiene que,

cuando el movimiento de la identidad asume, a través de portavoces, una
planificación pública de visibilización, de acción colectiva y efecto organizado en la
correlación de fuerzas del espacio social, puede materializarse como movimiento u
organización social permanente. Hablamos entonces de una identidad movilizada,
cuyo efecto social es capacidad de movilización. (p. 14)

No obstante, en lo referente al pueblo Mapuche, hablar de identidad es hacerlo también de territorio, no solo en consideración del histórico proceso de reducción al que se ha enfrentado sistemáticamente, sino también a la profunda conexión social, económica y espiritual que el pueblo Mapuche tiene con la tierra que ancestralmente ha habitado. En ese sentido, reconociendo el potencial de acción social que la identidad supone como mecanismo para la práctica de subjetividades compartidas, será importante reconocer el rol que el territorio, asumido más allá de sus características físicas (aunque si, también reconociendo su importancia), tiene no solo como espacio de construcción identitaria, sino también como elemento cohesionador de la comunidad consigo misma y con su entorno. En ese sentido, la relevancia conceptual del territorio constituido simbólicamente por quienes habitan en él, invita a la reflexión sobre cómo el sentido de pertenecía potencia el involucramiento individual y colectivo en procesos de participación en el ámbito territorial. En ese sentido, Bustos (2014) afirma que "considerando la importancia que tiene para una comunidad la supervivencia física, social y cultural, el territorio puede ser entendido como un espacio apropiado que permite a los sujetos y a la comunidad validar su desarrollo cultural. En ese sentido, el territorio termina siendo un mediador entre las diferentes esferas de la sociedad" (Bustos, 2014, p. $85)$. Esto, implica entonces un proceso de doble sentido dentro del cual los individuos son configurados identitariamente por el territorio en el que habitan, mientras que el mismo se configura a través de las subjetividades compartidas entre los individuos. A este mecanismo, Trejo (2008) lo define como apropiación, el cual,

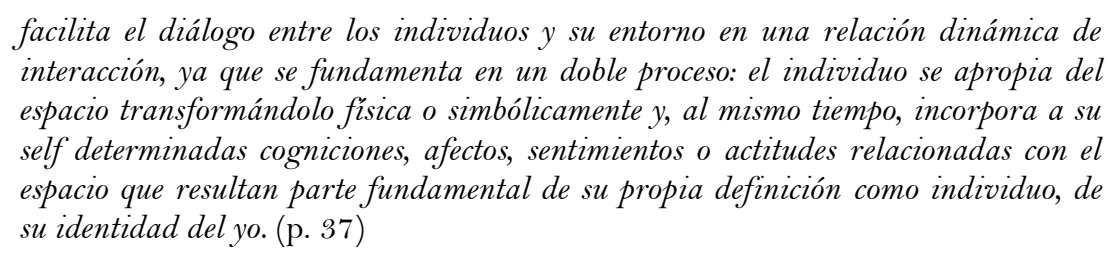

No obstante, es importante admitir que la construcción conceptual del territorio, no es una dinámica históricamente unilineal, sino un continuo y complejo dialogo entre el pasado, el presente y los posibles futuros. Por lo tanto, posicionándonos en los ámbitos educativo y comunitario, las transformaciones territoriales implican, por un lado; reconocer los saberes ancestrales o tradicionales de la comunidad y al mismo tiempo complementarlas con el conocimiento contemporáneo en busca de alternativas a los problemas territoriales (Bustos, 2014). Por otro lado, implica también la recuperación de la memoria colectiva, narrada por sus propias y propios protagonistas, ante la difusión uniforme de las narraciones históricas nacionales contenidas en los planes y programas oficiales.

Si bien, bajo los supuestos teóricos expuestos anteriormente, se asume una interrelación entre procesos educativos con las características descritas previamente, y el desarrollo de dinámicas de participación comunitaria a partir de la identidad movilizada y de la 
apropiación territorial, resultará fundamental acotar conceptualmente la noción y los ámbitos de participación, a fin de identificar sus principales características a partir de los casos estudiados. Peña, Almendra y Rojas (2010) señalan que,

el pueblo Mapuche, ancestralmente, ha usado un sistema de organización social y de identidad territorial muy característica y particular basado en un complejo sistema de alianzas y dependencias mutuas que tiene como base la identidad del territorio centrada en su linaje, esto es, en mapudungun el turwn ${ }^{3}$ y el kupalmet. (p. 249)

Lo anterior, advierte la existencia de

\begin{abstract}
un sistema político y organizativo que se afincaba principalmente en grupos familiares reducidos, lo cual le permite a los habitantes de una comunidad en particular, incorporar una forma única de relacionarse de los sujetos con un presente, pasado y futuro en esta relación de parentesco de cada familia y su comunidad. (Peña, Almendra y Rojas, 2010, p. 251)
\end{abstract}

Esta característica implica una forma de concebir los mecanismos y ámbitos de participación e incidencia, la cual podría asumirse como de pequeña escala o micropolítica, en referencia a territorios o comunidades concretas, en contraste, por ejemplo, con las características de la organización política del Estado Chileno, las cuales se basan en la defensa de los intereses generales de la nación, distribuyendo el poder político de forma centralista y cuya representación local puede ejercerse únicamente a través de los distintos mecanismos institucionales validados y organizados, como las elecciones o las consultas. No obstante, como se advirtió anteriormente, el hecho de que la estructura política institucional en Chile funcione central y verticalmente, no implica necesariamente que en paralelo no se gesten acciones concretas de participación social, ejercidas de forma territorial e impulsada por colectivos con objetivos bien delimitados. En ese sentido, la escuela, como muchos otros espacios de confluencia comunitaria, aparece como escenario en el cual, en consideración de sus características de interacción colectiva, pueden impulsarse diversos procesos de participación y fortalecimiento comunitario.

En consideración de todos estos antecedentes teóricos, es que se consideró necesario abordar empíricamente un conjunto de experiencias de vinculación entre comunidad y escuela, y a partir de ellas, definir cuáles son los factores que operan para promover dicho vínculo, lo cual constituye el objetivo primordial del presente texto y uno de los objetivos específicos del trabajo de investigación en el que se enmarca.

\title{
2. Hallazgos
}

Tal y como se señaló, los datos utilizados provienen de la cotidianeidad de tres escuelas municipales localizadas en las comunas de Contulmo y Tirúa, estudiada a través de la realización de entrevistas semiestructuradas con directivas y directivos, docentes, asesores y asesoras culturales, apoderadas y apoderados, alumnos y alumnas, autoridades locales o tradicionales y con colaboradores y colaboradoras del PEIB en la provincia de Arauco, quienes aportaron sus testimonios y experiencias en diversos procesos de vinculación entre la escuela y la comunidad. Considerando que se trató de una

\footnotetext{
${ }^{3}$ tuwvn s. origen geográfico del que se proviene, procedencia territorial; según el diccionario web del Instituto de lenguas y literaturas amazónicas (http://www.illa-a.org/wp/diccionarios/mapuche-wixaleyin/)

${ }^{4}$ kvpan s. 1 origen. 2 procedencia familiar (ídem)
} 
investigación de carácter cualitativo, no se buscó estandarizar la muestra, y la base de datos se configuró a partir de lo recopilado en ocho entrevistas individuales, 6 entrevistas colectivas y la observación participante en tres espacios sociales locales.

A partir de lo anterior, se logró identificar una serie de factores que permitirían promover y potenciar la vinculación participativa entre la escuela y la comunidad. Es importante mencionar que con esto no se pretende elaborar una suerte de "manual de buenas prácticas" utilizable en cualquier espacio, sino más bien subrayar aquellos puntos de confluencia en los que, a través de procesos diversos y particulares, las escuelas y comunidades participantes, coincidieron en señalar.

\subsection{Participación horizontal en el espacio escolar}

En primer lugar, cabe mencionar que, según los testimonios de las y los entrevistados, se destaca la importancia de la participación sostenida de actores y actrices comunitarias extra escolares, en las dinámicas organizadas dentro de la escuela, ya que su presencia legitima los esfuerzos de la misma, no solamente en actividades propiamente académicas, sino también en aquellas que se desarrollan de forma extracurricular. Lo anterior, no se reduce exclusivamente a la presencia de los actores arriba mencionados en el espacio escolar, sino que implica una redistribución de las responsabilidades en diversas actividades desarrolladas al interior de la escuela. En ese sentido, es importante mencionar que, en la gran mayoría de los casos, las y los entrevistados manifestaron que las relaciones entre el personal administrativo y docente, con el resto de la comunidad educativa se ha horizontalizado progresivamente, dejando atrás practicas jerárquicas y discriminatorias en la dinámica organizativa y participativa existentes hasta hace algunos años en las escuelas. En ese sentido, se considera que el involucramiento de las y los integrantes de la comunidad se implica en la decisión, ejecución y retroalimentación de las actividades cotidianas de los establecimientos educativos. Lo anterior se concreta en el hecho de que es la comunidad, muchas veces a través de grupos de apoderadas y apoderados, quienes han propuesto incluir al mapudungun como ramo oficial en las escuelas donde se desarrolló la investigación, y también en que es la propia comunidad, en este caso a través de las autoridades tradicionales, la que propone a la persona que desempeñara el cargo de educador tradicional, en el marco de la ejecución del PEIB. Por otro lado, en todas las escuelas se mencionó que las propuestas pedagógicas se someten antes a la aprobación de las y los apoderados, quienes también retroalimentan las mismas a través de lo que les mencionan sus hijas e hijos. Esto, permite que las acciones generadas en el ámbito escolar, no atraviesen únicamente aspectos académicos o curriculares sino que, al incluir la participación de diversos actores comunitarios, se proyecten como dinámicas de alcance territorial.

Sin embargo, sería ingenuo asumir que las experiencias positivas de vinculación entre la escuela y la comunidad se han gestado automáticamente, pues en todos los casos se trata de procesos derivados de la generación de lazos interpersonales entre las y los integrantes de la comunidad escolar, que trasciende lo estrictamente académico y apela a factores tales como las relaciones de parentesco y los sentidos comunitarios vigentes, los cuales generan entre las y los integrantes de la comunidad escolar sentidos de identidad colectiva territorializada. Al respecto, es importante resaltar que las profesoras consultadas coincidieron en asociar a la comunidad escolar con una familia y reiteradamente mencionan que sienten a las alumnas y alumnos como hijas o hijos, incluso dos de ellas mencionaron que el hecho de ser mujeres las dota de mayor 
sensibilidad e intuición para la contención colectiva y las posiciona como actrices de aglutinamiento colectivo. Es decir, las profesoras construyen su propio sentido de identidad a partir de la proyección misma de la acción educativa hacia y/o en referencia de la comunidad y sus referentes.

Otro de los aspectos relevantes en cuanto a la participación de actores comunitarios extraescolares, particularmente en lo referente a las apoderadas y apoderados, consiste en la divergencia manifiesta entre las escuelas participantes en cuanto a las características de matrícula de alumnos y alumnas de los establecimientos. Las comunidades escolares de las escuelas con menor número de estudiantes expresaron mayor fortaleza en cuanto a los lazos de colaboración dentro de las mismas, en contraste con aquellas que tienen una matrícula mayor que expresaron diversas dificultades para congregar, por ejemplo, a la totalidad de las y los apoderados en las actividades de la escuela por diversos motivos.

\subsection{Re significación de la escuela como punto de encuentro comunitario}

Por otro lado, en consideración de las características geográficas y espaciales de los sectores donde se localizan las escuelas participantes, se pudo constatar que éstas se han consolidado como un espacio de encuentro entre sus habitantes, en donde pueden no solo participar de actividades educativas, sino también abordar aspectos de relevancia para la comunidad, o simplemente ponerse al día en sus actualidades familiares. No obstante, lo anterior no solamente implica un atractivo para las y los apoderados, sino que también para las y los estudiantes quienes manifestaron que el espacio escolar se convierte en un punto de encuentro de sus amistades, donde incluso pueden desarrollar actividades que les son imposibles en otros ámbitos. En ese sentido, es importante destacar que para las niñas, niños y adolescentes del sector, las escuelas constituyen espacios de referencia dentro del territorio, ya que, como se mencionó anteriormente, este espacio se resignifica constantemente transformándose de un espacio institucional de enseñanza, en uno comunitario de socialización, entretenimiento y de practica cultural y deportiva. No obstante, el hecho de que los espacios escolares repliquen dinámicas comunitarias, no necesariamente implica una situación idílica en la relación entre las y los participantes, ya que muchas veces las relaciones de poder, o incluso los conflictos existentes dentro de las comunidades atraviesan y permean el espacio escolar.

En ese sentido, los espacios de convivencia informal dentro de las escuelas, no solamente funcionan como dinámicas de cohesión, sino que también se trasforman en espacios desestructurados de aprendizaje mutuo, lo cual revela la importancia del desarrollo de actividades extraescolares que involucren activamente a la comunidad, ya que durante estas se gestan dinámicas de construcción de conocimientos proyectados colectivamente y de forma colaborativa entre las y los participantes. Tal pareciera que los espacios informales contribuyen a difuminar las ya mencionadas jerarquías históricamente naturalizadas dentro de la institucionalidad educativa, en donde las y los docentes parecían poseer las únicas voces autorizadas para dirigir los procesos educativos y en general cualquier dinámica circunscripta al entorno escolar.

Aunque sería aventurado asumir que la resignificacion de los espacios escolares consisten en procesos íntegros de apropiación territorial, es importante reconocer que, como se ha mencionado en apartados anteriores, los propios espacios educativos se han transformado en espacios de articulación comunitaria en donde se reflexiona y se dialoga en torno a las reivindicaciones políticas, económicas y culturales vigentes en torno al 
territorio. Lo anterior, sin embargo, no implica que el espacio y la práctica educativa tenga que supeditarse necesariamente a las instalaciones de la escuela, ya que estas presentan importantes limitantes físicas y de disposición horaria para su uso. Por otro lado, como se señaló anteriormente, las escuelas son espacio de confluencia pero también de enfrentamiento, entre los ámbitos comunitario e institucional. La apropiación discursiva del territorio, manifiesta en espacios como el escolar, no debiese idealizarse como fin en sí mismo, ya que muchas y muchos de los entrevistados, coincidieron en destacar la importancia de la recuperación física de otros espacios dentro de su territorio, como los bosques o los cuerpos hídricos, en consideración no solo del aprovechamiento de sus recursos, sino también del valor simbólico que el mismo tiene para las comunidades Mapuche del sector.

\subsection{Abordaje integral del conflicto en el espacio escolar}

Es innegable que las comunidades localizadas en el cono sur de la Provincia de Arauco, se encuentran atravesadas por diversos conflictos sociales y territoriales presentes desde hace mucho tiempo en el sector. En ese sentido, uno de los principales intereses del presente artículo era indagar si los mismos eran un tópico que se aborda regularmente dentro del ámbito escolar y de qué forma se hace. A pesar de que los testimonios fueron diversos, fue posible advertir ciertas generalidades en el tipo de respuesta según los roles desempeñados dentro de las escuelas y en la comunidad. Por un lado, la mayoría de las directoras consultadas afirmaron que dentro de las escuelas no se abordaban las temáticas arriba descritas, con la intención de no generar dinámicas que pudiesen fragmentar a la comunidad escolar. No obstante, es importante mencionar que en todos los casos las directoras de los establecimientos participantes no están radicadas en los sectores donde se localizan las escuelas, por lo que, en contraste con los testimonios de las y los habitantes de las comunidades, manifestaron conocer de forma muy general la realidad de las mismas y por ende no estar al tanto de las circunstancias específicas que implican los conflictos dentro de las comunidades y como repercuten estas particularmente entre sus habitantes. Por el contrario, los educadores tradicionales, y/o profesoras avecindadas en los sectores donde se localizan las escuelas, señalaron que aunque conscientemente se procura no abordar temáticas coyunturales en lo respectivo la comunidad, estas aparecen continuamente en las escuelas por interés de las alumnas y alumnos, quienes, ya sea por la información que circula entre sus amigos o familiares, o a través de la que aparece en los medios de comunicación, se muestran intrigados sobre de los acontecimientos y también sobre las implicaciones individuales y comunitarias de los conflictos. Lo anterior, evidencia la importancia de abordar de forma crítica, no solo los acontecimientos recientes dentro de las comunidades, sino también cual ha sido el transito histórico del pueblo Mapuche, a fin de cuestionar y deconstruir una serie de estereotipos adjudicados al mismo desde diversos sectores, los cuales, según manifestaron algunos entrevistados, tienen notorias implicancias, particularmente en las niñas y niños, durante su proceso de construcción identitaria para asumirse o no como integrantes del pueblo Mapuche. En la misma tónica, algunas de las apoderadas entrevistadas manifestaron que las y los niños muestran natural curiosidad por los eventos desarrollados a partir de los conflictos territoriales y medioambientales, por la notoria presencia policiaca en diversos sectores del sur de la provincia de Arauco y por la participación de integrantes de sus familias en las movilizaciones. Considerando lo anterior, resulta evidente una divergencia discursiva entre los actores institucionales y los comunitarios al interior de las escuelas, en cuanto al tratamiento y relevancia que le 
tendría que ser asignada a los asuntos relacionados con los conflictos presentes dentro de las comunidades. Es importante particularizar que se asume que esta divergencia queda estrictamente en el plano de lo discursivo, porque según se pudo indagar a través de las entrevistas y la observación participante, los temas antes señalados son parte de la cotidianidad de las escuelas.

Sin embargo, a pesar de la renuencia de las y los actores institucionales para abordar integralmente los diversos conflictos manifiestos en el sector dentro de la escuela, paradójicamente, sus testimonios dieron cuenta de que se ha generado un cambio substancial que las docentes, quienes no son originarias del territorio, manifestaron experimentar en su percepción del mediáticamente denominado conflicto mapuche. Es decir, se enfrentan desde su experiencia a los diversos estigmas o estereotipos que sobre las comunidades admitieron poseer previamente a su participación en las escuelas. A pesar de que manifestaron no involucrarse activamente, las docentes consultadas se manifestaron críticas no solo con la excesiva presencia policial en la zona, sino también con la extensa presencia de cultivos forestales en consideración de sus impactos sociales y medioambientales. En ese sentido, y a pesar de que, como se mencionó anteriormente, las escuelas procuran no desarrollar debates sobre la temática forestal durante las clases, muchas de las y los alumnos entrevistados, principalmente las y los de $7^{\circ}$ y $8^{\circ}$, consideraron que la presencia de las plantaciones forestales ha significado el principal cambio negativo en el entorno, principalmente por su impacto medioambiental, particularmente en cuanto a la escasez de agua.

Por último, es importante mencionar que desde la diversidad de posturas valóricas e ideológicas presentes en los distintos casos estudiados, existen grupos de apoderados y apoderadas que consideran que en la escuela debiese hacerse un abordaje histórico y coyuntural de las temáticas relacionadas con el Pueblo Mapuche. En ese sentido, podría asumirse que la comunidad trasfiere hacia los espacios escolares, una de las tensiones existentes entre sus habitantes en cuanto a la reivindicación de la historia y el presente del pueblo Mapuche. Mientras que un sector de la comunidad asume que esta atraviesa únicamente por la revitalización de aspectos culturales como las ceremonias y la lengua, para otro, es también necesaria una reivindicación territorial y política del Pueblo Mapuche habitante del sector sur de la Provincia de Arauco. Ambos posicionamientos no son del todo contrarias, y se han traducido en posturas claras sobre los abordajes discursivos que del contexto se hacen dentro de la escuela, la cual, en voz de sus colaboradoras, se ha mantenido neutra ante los debates existentes en las comunidades.

\section{Conclusiones}

La escuela, como espacio de confluencia comunitaria, no está exenta de reproducir en su interior múltiples debates entre diversos sectores sociales presentes en la comunidad. Por lo anterior, sería ingenuo o idílico asumir que los espacios escolares operan exclusivamente mediante dinámicas armónicas para la transmisión o construcción del conocimiento, sino que por el contrario, estos procesos se generan muchas veces reproduciendo tensiones, conflictos y relaciones de poder, lo cual lejos de significar una dificultad insuperable para el proceso educativo, lo enriquece aportándole a las y los protagonistas del mismo de un marco para analizar la realidad críticamente y la posibilidad de generar acuerdos y estrategias de acción colectiva que trasciendan del ámbito educativo al comunitario. 
La educación bajo un enfoque comunitario, manifiesta en la participación activa dentro de la escuela, el enfoque de derechos humanos, manifiesto en el abordaje integral de los diversos conflictos que atraviesa el pueblo Mapuche, y la apropiación territorial, puesta en práctica a través de la resignificación de las escuelas, no son panaceas que por sí mismas resuelvan dificultades manifiestas cotidianamente en las escuelas y las comunidades, pero si son estrategias que permiten la apertura de espacios de diálogo y la construcción de territorios, ya que las mismas consideran en sus fundamentos conceptuales la posibilidad de que diversos grupos humanos puedan ejercer sus derechos en materia cultural, educativa, de participación y sobre el territorio, los cuales le han sido sistemáticamente negados al pueblo Mapuche.

Por último, asumiendo de antemano que es en el ámbito micropolítico donde se gestan progresivamente las transformaciones tangibles en el ámbito educativo en aspectos pedagógicos y de participación escolar, y que progresivamente se han ido recogiendo e incluyendo la participación de diversos actores comunitarios, es imperante asumir que el Estado Chileno, a través de estructura institucional y jurídica en materia educativas, no puede mantenerse al margen o estático ante las mismas. Es perentorio entonces acompañar estas experiencias de transformación, incorporando al diseño de política educativa perspectivas pedagógicas y de participación, que contribuyan a generar vínculos efectivos y duraderos entre las escuelas y las comunidades donde estas se localizan, a fin de legitimar un vínculo que día a día construyen y fortalecen las y los protagonistas del proceso educativo.

\section{Referencias}

Bustos, H. (2014). La importancia de la relación cultura, territorio y enseñanza de las ciencias. Enseñanza de las Ciencias y Cultura: Múltiples Aproximaciones, 7, 83-102.

Crespo, C. (2012) Buen vivir: Escenarios en disputa y nuevos sentidos para la educación. En F. Cevallos (Coord.), Educación y buen vivir: Reflexiones sobre su construcción (pp.63-78). Quito: Contrato Social por la Educación Ecuador.

Ferrão, M. (2010). Educación intercultural en América latina: Distintas concepciones y tensiones actuales. Estudios Pedagógicos, 36(2), 333-342.

García, A. (2014) Identidad Boliviana. Nación, mestizaje y plurinacionalidad. Vicepresidencia del Estado Plurinacional. La Paz: Asamblea Legislativa Plurinacional

Huircan, M. (2010). Desarrollo de la educación intercultural bilingüe en Chile. En D. Quilaqueo, C. Fernández y S. Quintriqueo (Eds.), Interculturalidad en contexto mapuche (pp. 19-41). Neuquén: Universidad Nacional del Comahue.

Instituto Nacional de Estadística. (2005). Estadísticas sociales de los pueblos indígenas en Chile. Censo 2002. Santiago de Chile: INE.

Llamas, P. (2014). ¿Es un fracaso la educación por competencias? Recuperado de http://www.lja.mx/2014/o6/es-un-fracaso-la-educacion-por-competencias-discere/

Luna, M. (2012) En busca del sentido de la educación ecuatoriana. En F. Cevallos, F. (Coord.), Educación y buen vivir: Reflexiones sobre su construcción (pp. 55-62). Quito: Contrato Social por la Educación Ecuador Mella, S. (2010). La reforma educacional de 1981 en la comunidad educativa de la provincia de Concepción. Concepción: Universidad de Concepción.

MINEDUC. (2011). PEIB-ORIGENES: Estudio sobre la implementación de la educación intercultural bilingüe. Santiago de Chile: Ministerio de Educación. 
Nogueira, H. (2008). El derecho a la educación y sus regulaciones básicas en el derecho constitucional chileno e internacional de los derechos humanos. Revista Ius et Praxis, 14(2), 209-269.

Peña, F., Almendra, O. y Rojas, M. (2010). Enfoque geográfico territorial de la educación intercultural. En D. Quilaqueo, C. Fernandez y S. Quintriqueo (Eds.), Interculturalidad en contexto mapuche (pp. 241-256). Neuquén: Universidad Nacional del Comahue.

Quintriqueo, S. y Cárdenas, C. (2010) Hacia una articulación entre conocimiento mapuche y conocimiento disciplinario en ciencia. En D. Quilaqueo, C. Fernández y S. Quintriqueo (Eds.), Interculturalidad en contexto mapuche (pp. 89-126). Neuquén: Universidad Nacional del Comahue.

Rojas, M. y Peña, F. (2015). Saberes ambientales lafkenche en escuelas de la costa de La Araucanía (Chile). Revista Latinoamericana de Ciencias Sociales, Niñez y Juventud, 13(2), 1207-1220.

Trejo, J. (2008). Habitar es narrativizar: El proceso de construcción de la identidad en los jóvenes vallechalquenses. Ciudad de México: Facultad Latinoamericana de Ciencias Sociales.

Venegas, H. (2012) Interculturalidad crítica como un aporte para el buen vivir. En F. Cevallos (Coord.), Educación y buen vivir: Reflexiones sobre su construcción (pp. 97-110). Quito: Contrato Social por la Educación

\section{Breve CV de los autores}

\section{Inti Torres Villegas}

Magister en Investigación Social y Desarrollo por la Universidad de Concepción. Becario de la Fundación W.K Kellogg para el Programa de Formación de Liderazgo en 2011 y para el Programa de Liderazgo Social en 2015. Colaborador durante más de siete años en diversas organizaciones no gubernamentales en el sur de México en temáticas relacionadas a la educación intercultural y a la participación de niñas, niños y adolescentes en comunidades mayas en el Estado de Yucatán. ORCID ID: 0000-00026011-1197. Email: intitorres@udec.cl

\section{Noelia Carrasco Henríquez}

Profesora Asociada de la Universidad de Concepción, Chile. Doctora en Antropología Social y Cultural por la Universitat Autónoma de Barcelona. Su línea de investigación son los estudios antropológicos del desarrollo, la antropología económica aplicada y la ecología política. Sus campos de trabajo etnográfico han sido los procesos de intervención y soberanía alimentaria, los relatos y las prácticas del desarrollo y la expansión de los monocultivos forestales en contexto de globalización capitalista. Imparte docencia en los campos de la antropología cultural, antropología económica y antropología aplicada, en programas de pre y post grado. ORCID ID: 0000-0001-55609866. Email: noeliacarrasco@udec.cl 